\title{
CORRECTION
}

\section{Correction to: Parametric Imaging of [11C]Flumazenil Binding in the Rat Brain}

Isadora Lopes Alves, ${ }^{1}$ David Vállez García, ${ }^{1}$ Andrea Parente, ${ }^{1}$ Janine Doorduin, ${ }^{1}$ Ana Maria Marques da Silva, ${ }^{2}$ Michel Koole ${ }^{3}$ Rudi Dierckx, ${ }^{1}$ Antoon Willemsen, ${ }^{1}$ Ronald Boellaard ${ }^{1}$

${ }^{1}$ Department of Nuclear Medicine and Molecular Imaging, University Medical Center Groningen, University of Groningen, Groningen, The Netherlands

${ }^{2}$ Laboratory of Medical Imaging, School of Physics, Pontifícia Universidade Católica do Rio Grande do Sul, Porto Alegre, Brazil

${ }^{3}$ Department of Nuclear Medicine and Molecular Imaging, KU Leuven, Leuven, Belgium

\section{Correction to: Mol Imaging Biol}

https://doi.org/10.1007/s11307-017-1098-2

The name of the second author was incorrectly cited and is corrected now, as displayed here. 